\title{
Improved Long-term Health Related Quality of Life after Islet Transplantation
}

\author{
Thipaporn Tharavanij ${ }^{1,2}$, Arthur Betancourt ${ }^{3}$, Shari Messinger ${ }^{1,4}$, Pablo Cure ${ }^{1}$, Cristiane B. \\ Leitao $^{1}$, David A. Baidal ${ }^{1}$, Tatiana Froud ${ }^{1,5}$, Camillo Ricordi ${ }^{1,5}$, and Rodolfo Alejandro ${ }^{1,6}$ \\ ${ }^{1}$ Diabetes Research Institute, University of Miami, Miami, Florida 33136, USA. ${ }^{2}$ Department of \\ Medicine, Thammasat University, Pratumthani, Thailand. ${ }^{3}$ Departments of Psychiatry and \\ Behavioral Science, University of Miami, Miami, Florida, USA. ${ }^{4}$ Department of Epidemiology and \\ Public Health ${ }^{5}$ Department of Surgery, 1450 NW 10th AVE, Miami FL 33136 USA ${ }^{6}$ Department of \\ Medicine
}

\begin{abstract}
Background-Health related quality of life (HRQoL) is one of the most important outcomes to measure effectiveness of an intervention, especially for islet transplantation in which benefits should outweigh risks of long-term immunosuppression. This study aimed to evaluate long-term effects of islet transplantation and to outline possible influential factors.

Methods-Forty islet transplant recipients who completed 344 Health Status Questionnaires (HSQ 2.0) and 384 Diabetes quality of life questionnaires (DQoL) between 2000-2007 were retrospectively reviewed. Assessments were analyzed in pre-transplantation period, then every 3 months after the first infusion for 18 months and every 6 months thereafter. The mean follow-up post-transplantation was $40.8 \pm 21.9$ (9-72) months.
\end{abstract}

Results-Sustained improvement in DQoL-impact score was observed at all time-points posttransplantation. Similarly, worry and satisfaction scales were significantly better than pre-transplant evaluation for most time-points. Four-out-of-eight HSQ 2.0 scales demonstrated a significant improvement at some time-points. Longitudinal analysis, after adjustments for potential confounding factors, showed significantly sustained improvement in impact scale up to 72 months. Longer diabetes duration, higher insulin dosage and occurrence of adverse events had negative effects on HRQoL. Single islet infusion or islet after kidney transplant recipients showed the lowest values in HSQ 2.0. In contrast, subjects on exenatide therapy had significantly higher HSQ 2.0 scores.

Conclusions-Islet transplantation is associated with long-term improvement in HRQoL. Exenatide usage had a positive effect while single islet infusion, islet after kidney transplantation, longer diabetes duration, higher insulin dosage and adverse events had a negative impact on HRQoL scores.

\section{Keywords}

quality of life; islet transplantation; diabetes

Address for Correspondence: Rodolfo Alejandro MD, Diabetes Research Institute, 1450 NW 10th Avenue (R-134), Miami, FL 33136 USA, ralejand@med.miami.edu, Tel (305) 243-5324; Fax (305) 243-1058.

None of the authors had a personal or financial conflict of interest. 


\section{Introduction}

Islet transplantation is a therapeutic option for patients with type 1 diabetes mellitus with unstable metabolic control (1-3). Despite the tendency for graft dysfunction overtime $(1,2,4)$, a significant improvement in Diabetes Quality of life (DQoL) has been shown in islet transplant recipients, up to 36 months post completion (5). In addition, better Hypoglycemia Fear Survey scores after islet transplantation were observed in two cohorts $(6,7)$. However, islet transplantation had none or minimal effects on generic health related quality of life (HRQoL) evaluated by the Health Status Questionaires (HSQ 2.0), Health Utility Index Mark 2 (HUI2) and 36-Item Short Form Health Survey (SF-36) (5-7). This could be explained by the use of generic HRQoL questionnaires, which provide more information about functional health status. Disease-specific HRQoL such as DQoL is known to be more sensitive in recording the negative effects arising from diabetic complications or changes after intervention (8-10). Insulin requirements, number of islet infusions and glucose stability had an impact on HRQoL after islet transplantation $(5,6)$, however a systematic evaluation of factors affecting the HRQoL are still lacking.

It is important that the benefit of islet transplantation should outweigh the risks of long-term use of immunosuppressive agents. HRQoL is another way to assess islet transplantation success. Until now, there has been no data evaluating long-term HRQoL after islet transplantation. Based on previous studies demonstrating short term improvement in HRQoL after islet transplantation (5-7), we hypothesized whether this same outcome could also be achieved after long-term follow up. The present study is an extension of the previous one (5) with a larger sample and longer follow-up, in order to explore long-term HRQoL and its influential factors in type 1 diabetes subjects after islet transplantation.

\section{Materials and Methods}

Islet transplant recipients from 2000-2007 (4,11-13), who had HRQoL data available were retrospectively reviewed. A total of forty out of 44 recipients (19 males and 21 females) performed self-completed HRQoL questionnaires. All subjects were white and most of them were nonHispanic except one. Seven subjects graduated from high school, twelve attended college, fourteen finished associate/bachelor degree and seven had post-college graduate degree. These subjects underwent islet transplantation alone (IA) $(n=26)$, islet after kidney transplant (IAK) $(n=7)$ or islet with bone marrow transplant (IBM) $(n=7)$. The pancreatic islet isolation, infusion and immediate post-transplant management were performed as previously described (4). Patients received $1(n=15), 2(n=18), 3(n=6)$ or $4(n=1)$ islet infusions. The decision of receiving single islet infusion was based on the protocol, patient request, poor compliance, side effects or immunological sensitization. Maintenance immunosuppressive regimen was based on sirolimus (Rapamune ${ }^{\circledR}$, Wyeth Pharmaceuticals, Inc., Madison, NJ, USA) and tacrolimus (Prograf ${ }$, Astellas-Pharma US, Inc., Deerfield, IL, USA). Eleven subjects switched from tacrolimus/sirolimus to mycofenolate mofetil or mycofenolate sodium due to adverse events $(n=8)$, per protocol $(n=2)$ or patient decision $(n=1)$. Immunosuppressive drugs were discontinued in two subjects due to infection. Twenty-one subjects had received exenatide (Byetta ${ }^{\circledR}$, Amylin Inc.) (mean 17 \pm 10 months, from 0.6-28.6 months) in order to improve graft function and/or islet engraftment (phase II study).

Both generic and disease-specific questionnaires were used as instruments to evaluate HRQoL. The generic health status was measured by the Health Status Questionnaire (HSQ 2.0), which reflects the health burden of chronic disease and the effect of treatments on general health status. It is adapted from SF-36 with the same questions and scoring algorithm except for bodily pain. HSQ 2.0 also includes the screening for dysthymia, major depression, index of health status change and demographic items to assess factors associated with functioning and well- 
being. This instrument has been validated with good consistency and reliability (14). HSQ 2.0 has 8 scales: health perception, physical functioning, role limitation-physical health, role limitation-emotional problems, social functioning, mental health, bodily pain and energy/ fatigue. Disease-specific quality of life was assessed by DQoL which is designed to have 3 subscales to measure satisfaction of treatment, impact of therapy, worry about diabetes/social and vocational aspects. This questionnaire has been previously validated in type 1 diabetes (15). Both assessments have scores in each scale range 0-100 (from the worst to the best).

All subjects completed both questionnaires face to face at our clinic as a part of general psychological evaluation. The frequency of follow-up evaluation was designed to allow subjects the opportunities to report on their current experiences and perception based on timing of insulin independence, graft dysfunction and occurrence of adverse events (2-4). The time points analyzed were as follows: baseline (pre-transplantation), every 3 months after the first infusion for 18 months and every 6 months thereafter. Pre-transplant time-points were also reviewed to investigate significant changes of $\mathrm{HRQoL}$ before transplant and this data was used as control. The questionnaires were grouped into 0-6, 6-12 and >12 months before islet transplantation. In addition, all subjects were asked the same open-ended questions to explain the most important benefit from islet transplantation, the impact of that benefit and whether they have any regrets participating in a clinical trial. Answers were recorded verbatim.

Baseline characteristics (eg. age, sex, duration and complications of diabetes), number of islet infusions, islet transplant protocol (IA, IAK or IBM), glucose control (A1C), insulin dosage, exenatide administration and adverse events pre and post-transplantation were recorded. The adverse events were classified according to Common Terminology Criteria for Adverse Events, version 3.0 (National Cancer Institute; NCI) and categorized as NCI I (mild), II (moderate), III (severe) and IV (life-threatening). All protocol procedures were approved by the University of Miami health research ethics board and appropriate informed consent was obtained from each subject.

\section{Statistical analysis}

Statistical analysis and graphics were done using Excel® for Windows ${ }^{\circledR}$, SAS 9.1 (SAS Institute Inc., Cary, NC, USA) and SSPS 15.0 software. Data analyses were performed by using appropriate methods to the longitudinal nature. For each of the $11 \mathrm{HRQoL}$ scale measurements under consideration, we performed repeated measure analysis by using linear mixed model regression. This method of analysis generalizes linear regression techniques to allow for repeated observations by taking into account the correlation that exists within observations on the same subject in estimating variances used for the various tests of significance. Using this approach, we are able to simultaneously estimate differences between baseline and each time point post-islet transplantation. We additionally used this approach to estimate bivariate associations between each HRQoL outcome and each potential explanatory factor considered in this research including age, diabetes duration, number of islet infusion, recent period ( 3 months) after each infusion, insulin dosage, A1C, protocol, exenatide usage and adverse events. A more formal analysis of the data was then performed by incorporating these factors into the regression models for the effect of time, in order to provide adjustment for potential confounding. This analysis included data collected pre-transplant and 14 time-points posttransplant up to 72 months. Missing data was handled with pairwise deletion analysis (16). Baseline characteristics were expressed in mean \pm SD. The model-based estimate concerning HRQoL scores were expressed in mean \pm SEM. Differences were considered statistically significant at a $p$ value $<0.05$.

Transplantation. Author manuscript; available in PMC 2009 November 15. 


\section{Results}

Forty subjects answered 728 questionnaires which comprised 344 HSQ 2.0 and 384 DQOL. Mean follow-up pre and post-transplant were 23.2 $\pm 22.6(1-106)$ and $40.8 \pm 21.9$ (9-72) months, respectively. The mean age at the time of the first questionnaires obtained was $41 \pm 8.5$ years old. Diabetes duration ranged from 5-45 years (mean 26.8 \pm 11.8 ). Chronic diabetes complications at baseline included retinopathy $(\mathrm{n}=27)$, nephropathy $(\mathrm{n}=12)$, neuropathy $(\mathrm{n}=16)$, hypertension $(\mathrm{n}=12)$ and stable coronary artery disease $(\mathrm{n}=1)$. At the time of data analysis, 5 subjects reached 72-month post-transplant follow-up. Data was available for only $29.9 \pm 19$ (9-66) months post-transplantation in sixteen subjects due to: loss of follow-up ( $\mathrm{n}=1)$, side effects $(n=4)$, graft loss $(n=3)$, as per protocol $(n=4)$ in IBM recipients (13) and patient decision $(n=4)$. Data completeness was achieved on $86.8 \%$ of the post-transplant time points.

Seventy percent of the recipients reached insulin independence. At the end of the evaluation, 7 patients (17.5\%) were still insulin independent. A decrease in mean insulin requirements from $0.45 \pm 0.16(0.13-0.8)$ to $0.12 \pm 0.16(0-0.76) \mathrm{IU} / \mathrm{kg} / \mathrm{day}(\mathrm{p}<0.001)$ and in A1C from 7.5 \pm 1.1 to $6.2 \pm 0.8 \%$ ( $\mathrm{p}<0.001$ ) were observed when all pre-transplant were compared with posttransplant time-points.

\section{DQoL post-islet transplantation}

Impact scores were higher at all post-transplant time-points in comparison with pretransplantation. Worry scale showed a significant improvement except in the first 3 months after first infusion. A significantly increase in satisfactory score was observed at most timepoints, except at 3, 30-42 and 72 months (Table 1).

\section{HSQ 2.0 post-islet transplantation}

A significant increase in HSQ 2.0 scales; health perception, physical functioning, social functioning and bodily pain was observed at some time points (Table 1). In contrast, there was a transient decrease of role limitation-physical health, role limitation-emotional problem and mental health at various time points.

\section{Pre-transplant questionnaire evaluation}

The analysis of HRQoL comparing 0-6 months $(\mathrm{n}=23)$ with $>12$ months $(\mathrm{n}=33)$ pretransplantation, showed significant increases in DQoL [satisfaction (80 \pm 2 vs $69 \pm 3$ at $0-6$ and $>12$ months pre-transplantation respectively, $\mathrm{p}=0.016)$ ] and HSQ [energy scales $(79 \pm 4$ vs 70 $\pm 3, \mathrm{p}=0.029)]$.

\section{Factors influencing HRQoL}

Diabetes duration, transplant protocol, number of islet infusions, insulin dosage, adverse events and exenatide administration influenced various scales of HRQoL (Table 2). IAK recipients had the lowest scores in many HSQ 2.0 scales in comparison with those from other protocols. Single islet infusion recipients, whose estimate mean insulin requirement was $0.14 \mathrm{IU} / \mathrm{kg} / \mathrm{day}$ higher than recipients of 2 or more infusions ( $\mathrm{p}=0.003$ ), had worse HSQ 2.0 scores (health perception, role limitation-emotion, social function and energy scales) than multiple infusion recipients. To evaluate if a recent islet infusion had negative effect on quality of life, the scores available within 3 months after each infusion were compared with the other time-points and no significant difference in HRQoL, except for improvement of worry scale ( $\mathrm{p}=0.04)$, was observed.

Subjects on exenatide had higher HSQ 2.0: health perception and mental health scores and less NCI III-IV events $(\mathrm{OR}=0.4,95 \% \mathrm{CI} 0.17-0.93)$ than those without exenatide. Insulin requirement and $\mathrm{A} 1 \mathrm{C}$ between these 2 groups was not different $(\mathrm{p}>0.05)$. From the interviewing 
aspect, they believed that exenatide would prolong islet graft survival and stabilize blood glucose control.

Higher A1C had a trend toward worse scores of most HRQoL scales without reaching statistical significance (Changes of scores ranged from $0.99 \pm 0.7$ to $-3.1 \pm 4.1$ for every $1 \%$ increase of A1C, $p>0.05)$. Age and specific diabetic complications did not influence any HRQoL scales.

After adjusting for confounding factors, there was an improvement of HSQ 2.0: role-limitation emotion at 9-12 months, bodily pain at 72 months and energy scale at 6 and 18 months posttransplantation $(\mathrm{p}<0.05)$. However, no significant decrease of the score was observed.

Sustained improvement in DQoL was detected especially for worry and impact scales up to 60 and 72 months, respectively (Fig. 1).

From the interview data, all subjects responded that having stable blood glucose control and not being afraid of having a hypoglycemic episode was the most important benefit after transplantation which led to the feeling of independence. No subject responded any regrets for transplantation. Moreover they had pleasure to be a part of the research.

\section{Discussion}

HRQoL is one of the most important outcomes to measure effectiveness of an intervention. Improvement in HRQoL is associated with better diabetes self-management and lifestyle modifications (17). In contrast, a decrease in HRQoL can have a negative impact on patient compliance (8). This study combined generic and disease-specific instruments to include important aspects that could have an impact on HRQoL.

The present study, with a longer follow-up and a larger sample size than the previous publications $(5,7)$, demonstrated that the improvement of HRQoL was maintained up to 6 years after islet transplantation. DQoL in both unadjusted and adjusted analysis were clearly associated with significant improvement in all scales at most time-points. HSQ 2.0 failed to demonstrate the consistency of improvement after transplantation. This may be due to the subject attrition and also because this instrument focuses on more general aspects of health perception. A transient decrease in role limitation-emotional problem, role limitation-physical health and mental health was detected but it was not sustained after adjustments for confounding factors.

Our results are different from the Diabetes Control and Complications Trial (DCCT), which did not find any improvement in both generic and disease specific HRQoL after intensive insulin therapy when comparing with conventional treatment (18). The stable metabolic control, insulin independence or less insulin requirements after islet transplantation may have lead to a greater improvement in HRQoL than intensive insulin therapy. However, subjects selected for islet transplantation had more unstable diabetes than those in the DCCT trial, which could lead to more obvious change in HRQoL after intervention.

Longer diabetes duration, higher insulin dosage and the occurrence of adverse events had significant negative effects on HRQoL. Our observations were similar to those from previous reports, in which worse HRQoL was associated with insulin usage $(5,9)$. In contrast, higher A1C had a trend toward worse HRQoL but without statistical significance. This result was different from prior studies (19-20). Lack of significant changes might be due to stable glycemic control over time after islet transplantation.

Insulin independence after islet transplant is usually achieved after at least two islet infusions (2). Our study revealed that subjects who received only a single islet infusion had lower generic quality of life scores. Higher insulin requirements in this subgroup might be responsible for

Transplantation. Author manuscript; available in PMC 2009 November 15. 
this observation. Also, after comparing different protocols, IAK recipients had the worst scores in most scales of HSQ 2.0, which might be explained by a more severe nature of the underlying disease.

Exenatide usage had positive effects on mental health and health perception scales of HSQ 2.0. The association of exenatide with a significantly lower rate of severe adverse events was unexpected. The latter might explain the benefits on quality of life after exenatide initiation. In addition, patients anticipated that exenatide would maintain or improve islet function and stabilize glucose control.

Most of post-transplant adverse events were procedure or medication-related and the higher incidence was during the first 3 months after infusion (4). We did not find any differences when comparing the HRQoL at 3 months post infusion (regardless the number of infusions) with the other time-points, except for a better worry score at 3 months post infusion period. This finding was similar to a previous study, which reported better emotion scores, evaluated by HUI2 after the first islet infusion (6).

The limitation of this study was the lack of a control group. In order to overcome this issue, pre-islet transplantation data $(\mathrm{n}=68)$ were analyzed. Since subjects underwent HRQoL evaluation in more than one time-point before transplant, the comparison between pretransplant results were done in order to evaluate the HRQoL changes before intervention. Even though, two out of total $11 \mathrm{HRQoL}$ scales showed significant improvement before transplantation, the consistently better DQoL results maintained up to 6 years, indicate that improvement in HRQoL was most likely due to the islet transplantation effect. In addition, the verbal interview confirmed that the recipients were satisfied with the stability of their glucose control and absence of severe hypoglycemia. Subjects also reported a feeling of independence and being pleased with their participation in the clinical trial.

Another study limitation could have been the study withdrawals which could have positively affected the final outcome such as only patients benefited in the trial participated until the end of the study. In the future, randomized clinical trials aiming to compare HRQoL after islet transplantation with either pancreatic transplantation or intensive insulin therapy in unstable type 1 diabetes as a control group should be conducted.

In conclusion, a consistent long-term improvement in HRQoL after islet transplantation reflecting by DQoL was observed. Exenatide usage had a positive effect on quality of life, whereas longer diabetes duration, islet after kidney transplantation, single islet infusion, adverse events occurrence and higher insulin dosage had negative impact on health related quality of life.

\section{Acknowledgements}

The authors are grateful to the staff of the Clinical Islet Transplant Program, Islet Cell Processing Center, General Clinical Research Center for the continuous support.

The authors' responsibilities were as follows-TT, CBL, TF, RA: study design; TT, AB, PC, DAB, TF: data collection; TT, SM: analysis of data; every authors contributed to writing the manuscript; and CR, RA: provision of significant advice.

This study was supported by: NIH-National Center for Research Resources (U42 RR016603, M01RR16587); NIDDK (5R01-DK55347, 5R01-DK056953, R01-DK025802, 1RO1-DK25802-21; 1RO1-D59993-04); JDRF International (4-2000-946 and 4-2004-361); State of Florida; and Diabetes 


\section{Abbreviation}

HRQoL, health related quality of life; HSQ 2.0, Health Status Questionnaires; DQoL, Diabetes quality of life questionnaires; HUI2, Health Utility Index Mark 2; SF-36, 36-Item Short Form Health Survey; IA, islet transplantation alone; IAK, islet after kidney transplant; IBM, islet with bone marrow transplant; NCI, National Cancer Institute; ITx, islet transplantation.

\section{References}

1. Shapiro AM, Ricordi C, Hering BJ, et al. International trial of the Edmonton protocol for islet transplantation. N Engl J Med 2006;355:1318. [PubMed: 17005949]

2. Ryan EA, Paty BW, Senior PA, et al. Five-year follow-up after clinical islet transplantation. Diabetes 2005;54:2060. [PubMed: 15983207]

3. Ricordi C. Islet transplantation: a brave new world. Diabetes 2003;52:1595. [PubMed: 12829621]

4. Froud T, Ricordi C, Baidal DA, et al. Islet transplantation in type 1 diabetes mellitus using cultured islets and steroid-free immunosuppression: Miami experience. Am J Transplant 2005;5:2037. [PubMed: 15996257]

5. Poggioli R, Faradji RN, Ponte G, et al. Quality of life after islet transplantation. Am J Transplant 2006;6:371. [PubMed: 16426323]

6. Toso C, Shapiro AM, Bowker S, et al. Quality of life after islet transplant: impact of the number of islet infusions and metabolic outcome. Transplantation 2007;84:664. [PubMed: 17876283]

7. Barshes NR, Vanatta JM, Mote A, et al. Health-related quality of life after pancreatic islet transplantation: a longitudinal study. Transplantation 2005;79:1727. [PubMed: 15973176]

8. Bradley C. Importance of differentiating health status from quality of life. Lancet 2001;357:7. [PubMed: 11197385]

9. Jacobson AM, de Groot M, Samson JA. The evaluation of two measures of quality of life in patients with type I and type II diabetes. Diabetes Care 1994;17:267. [PubMed: 8026281]

10. Jacobson, AM. the DCCT research group. The Diabetes Quality of Life Measure. In: Bradley, C., editor. Handbook of psychology and diabetes: a guide to psychological measurement in diabetes. Harwood: Chur; 1994. p. 65

11. Cure P, Pileggi A, Froud T, et al. Improved metabolic control and quality of life in seven patients with type 1 diabetes following islet after kidney transplantation. Transplantation 2008;85:801. [PubMed: 18360260]

12. Froud T, Faradji RN, Pileggi A, et al. The use of exenatide in islet transplant recipients with chronic allograft dysfunction: safety, efficacy and metabolic effects. Transplantation 2008;86:36. [PubMed: 18622276]

13. Mineo D, Ricordi C, Xu X, et al. Combined islet and hematopoietic stem cell allotransplantation: a clinical pilot trial to induce chimerism and graft tolerance. Am J Transplant 2008;8:1262. [PubMed: 18444924]

14. Stewart ALGS, Hays RD, Wells K, Rogers WH, Berry SD, McGlynn EA, Ware JE Jr. Functional status and well-being of patients with chronic conditionsResults from the Medical Outcomes Study. JAMA 1989;262:907. [PubMed: 2754790]

15. Reliability and validity of a diabetes quality-of-life measure for the diabetes control and complications trial (DCCT). The DCCT Research Group. Diabetes Care 1988;11:725. [PubMed: 3066604]

16. Reoffrey, RN.; David, LS. Screwups, oddballs, and other vagaries of science. Locating outliers, handling missing data, and transformations. In: Geoffrey, RN.; David, Ls, editors. Biostatistics. Hamilton: BC Decker Inc; 2008. p. 302

17. Zhang X, Norris SL, Chowdhury FM, Gregg EW, Zhang P. The effects of interventions on healthrelated quality of life among persons with diabetes: a systematic review. Med Care 2007;45:820. [PubMed: 17712252]

18. Influence of intensive diabetes treatment on quality-of-life outcomes in the diabetes control and complications trial. Diabetes Care 1996;19:195. [PubMed: 8742561]

19. Hoey H, Aanstoot HJ, Chiarelli F, et al. Good metabolic control is associated with better quality of life in 2,101 adolescents with type 1 diabetes. Diabetes Care 2001;24:1923. [PubMed: 11679458]

Transplantation. Author manuscript; available in PMC 2009 November 15. 
20. Guttmann-Bauman I, Flaherty BP, Strugger M, McEvoy RC. Metabolic control and quality-of-life self-assessment in adolescents with IDDM. Diabetes Care 1998;21:915. [PubMed: 9614607] 


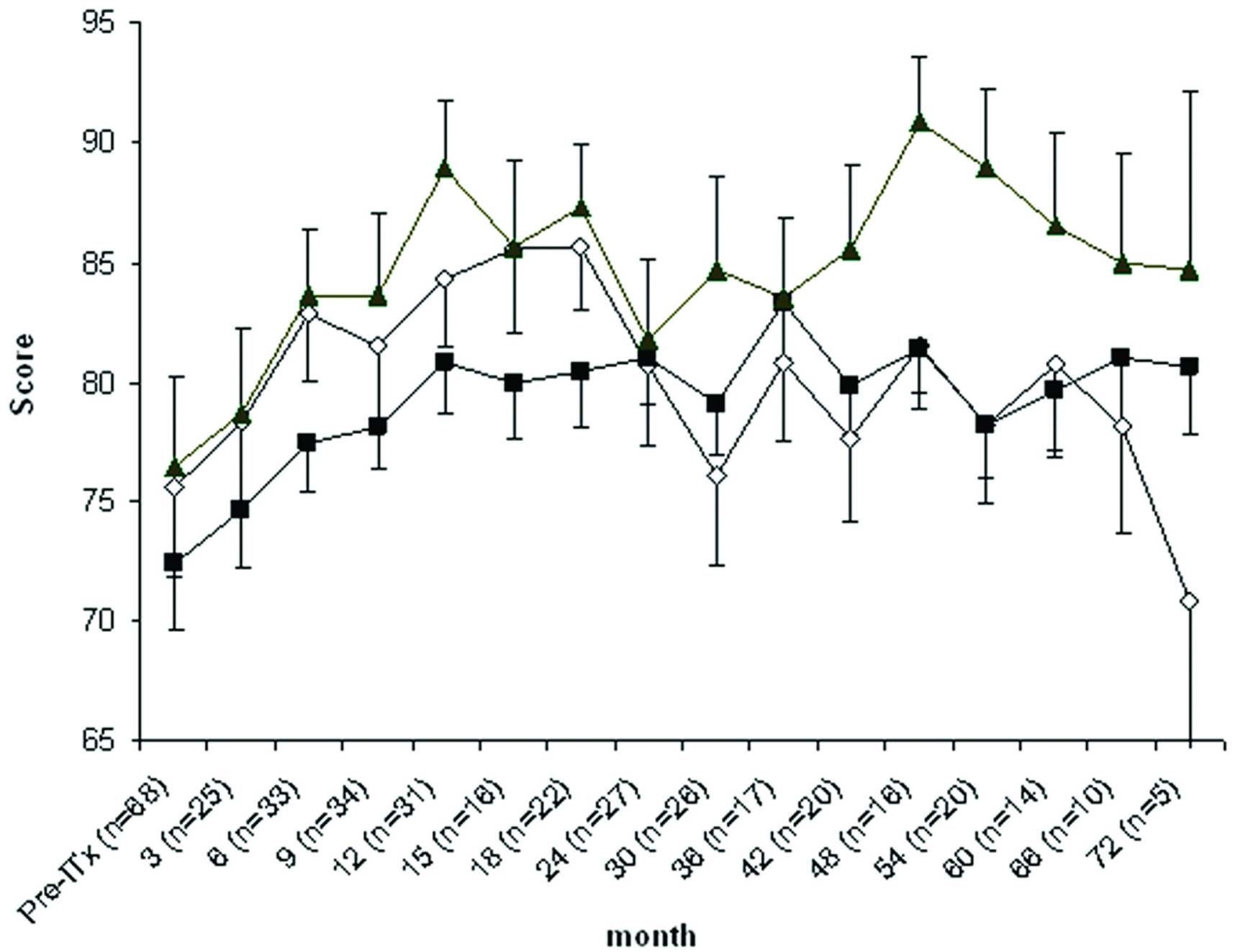

Figure 1.

Diabetes quality of life (DQoL) before and after islet transplantation (ITx) after adjusting for confounding factors. Data are mean estimate \pm SEM. Comparing with pre-ITx, satisfaction scale (open diamond) had significant improvement at 6 and 12-18 months after transplant. Impact scale (closed square) was significantly improved at 9-48 and 60-72 months. Worry scale (closed triangle) had significantly higher scores at 6-18 and 30-60 months $(\mathrm{p}<0.05)$. 


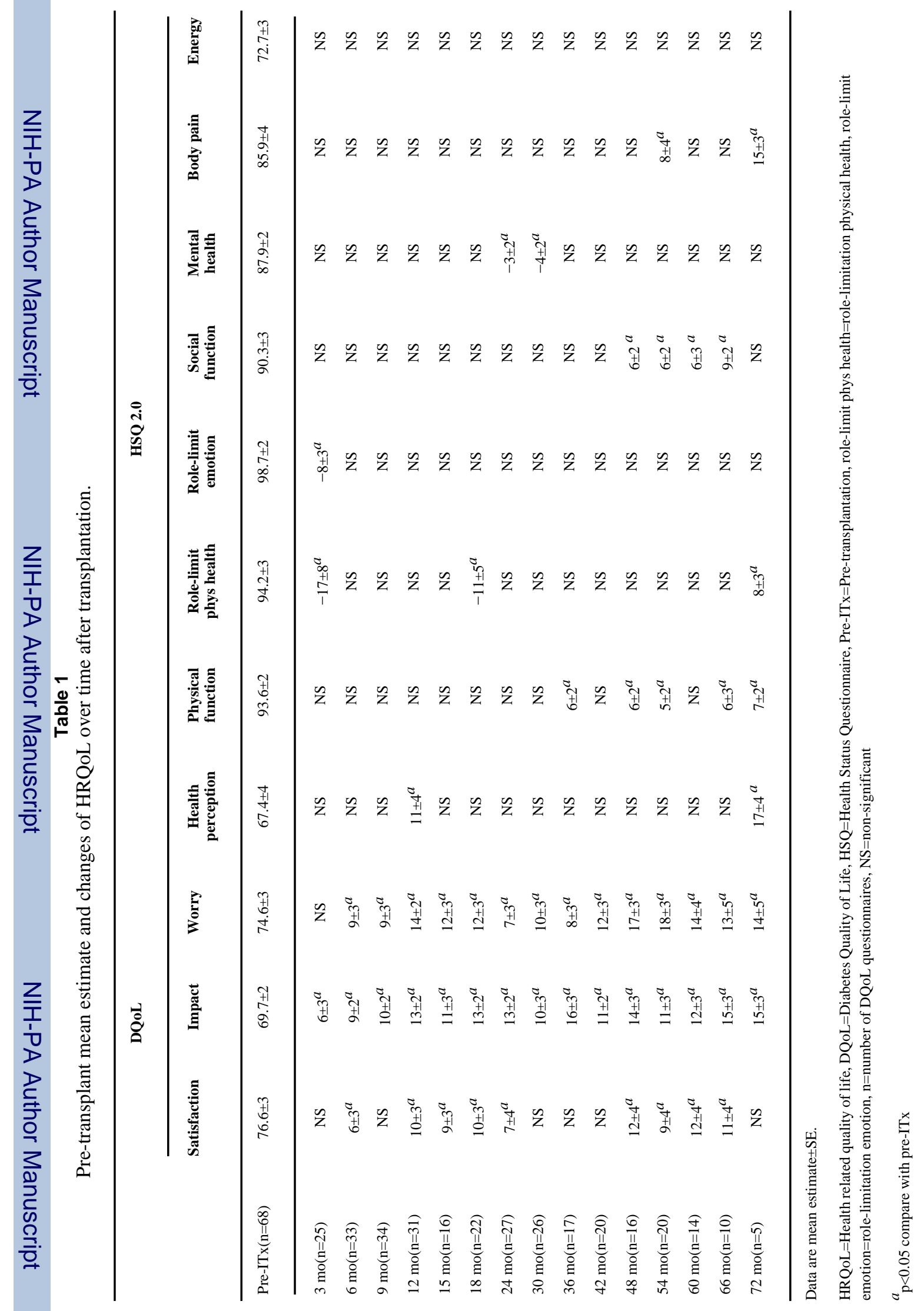




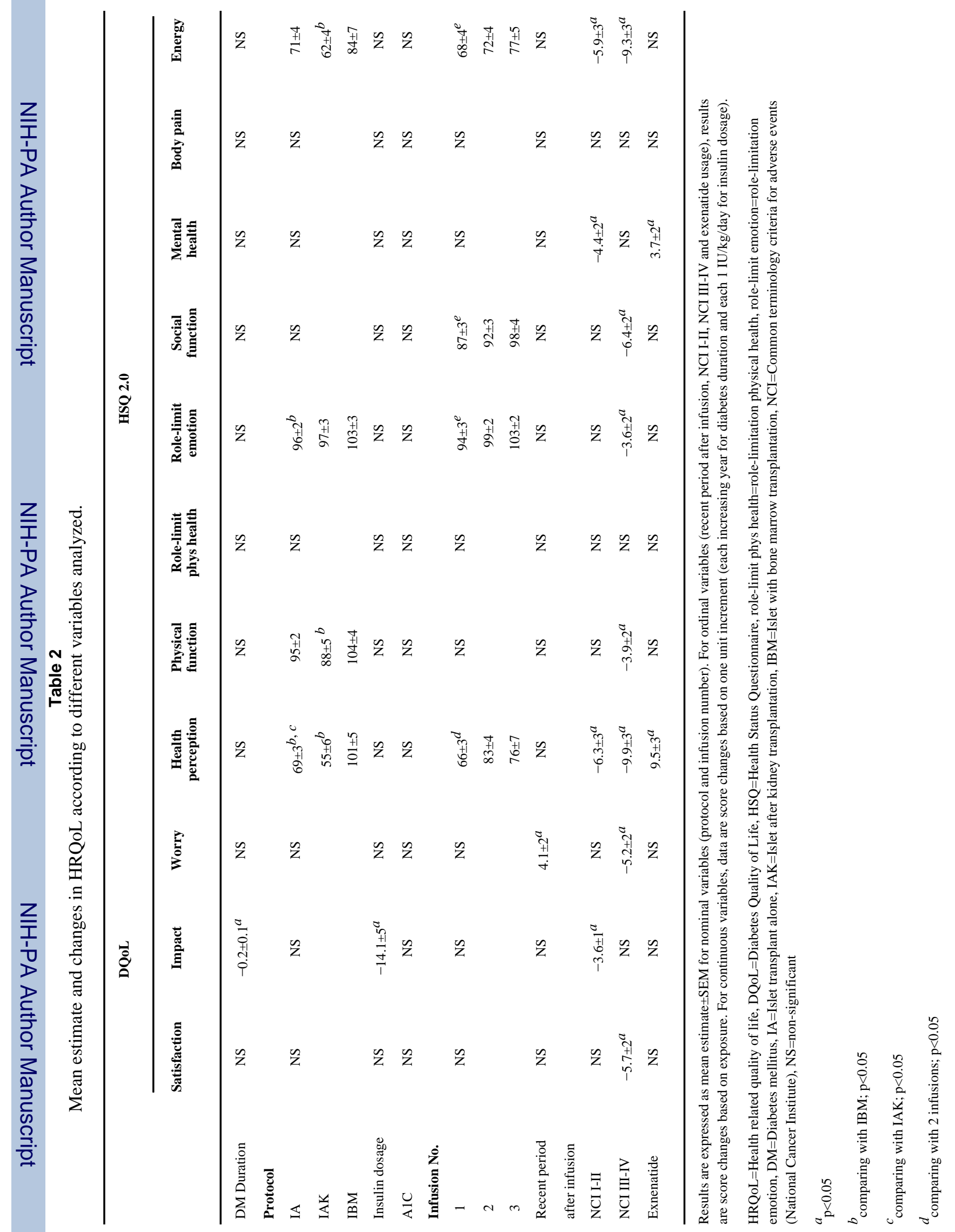
Transplantation. Author manuscript; available in PMC 2009 November 15. 
